\title{
Does standard cosmology really predict the cosmic
}

\section{microwave background? [version 1; peer review: 1 not}

\section{approved]}

\section{Hartmut Traunmüller (iD}

Department of Linguistics, Stockholm University, Stockholm, SE-106 91, Sweden

V1 First published: $16 \mathrm{Apr} 2020,9: 261$

https://doi.org/10.12688/f1000research.22432.1

Second version: 03 Jun 2020, 9:261

https://doi.org/10.12688/f1000research.22432.2

Third version: 07 Jul 2020, 9:261

https://doi.org/10.12688/f1000research.22432.3

Fourth version: 28 Sep 2020, 9:261

https://doi.org/10.12688/f1000research.22432.4

Fifth version: 19 Feb 2021, 9:261

https://doi.org/10.12688/f1000research.22432.5

Latest published: 23 Sep 2021, 9:261

https://doi.org/10.12688/f1000research.22432.6

\section{Abstract}

In standard Big Bang cosmology, the universe expanded from a very dense, hot and opaque initial state. The light that was last scattered about 380,000 years later, when the universe had become transparent, has been redshifted and is now seen as thermal radiation with a temperature of $2.7 \mathrm{~K}$, the cosmic microwave background (CMB). However, since light escapes faster than matter can move, it is prudent to ask how we, made of matter from this very source, can still see the light. In order for this to be possible, the light must take a return path of the right length. A curved return path is possible in spatially closed, balloon-like models, but in standard cosmology, the universe is "flat" rather than balloon-like, and it lacks a boundary surface that might function as a reflector. Under these premises, radiation that once filled the universe homogeneously cannot do so permanently after expansion, and we cannot see the last scattering event. It is shown that the traditional calculation of the CMB temperature is flawed and that light emitted by any source inside the Big Bang universe earlier than half its "conformal age", also by distant galaxies, can only become visible to us via a return path. Although often advanced as the best evidence for a hot Big Bang, the CMB actually tells against a formerly smaller universe and so do the most distant galaxies. While standard cosmology has additional deficiencies, those disclosed here defy rationality and therefore make a more well-founded cosmology indispensable.

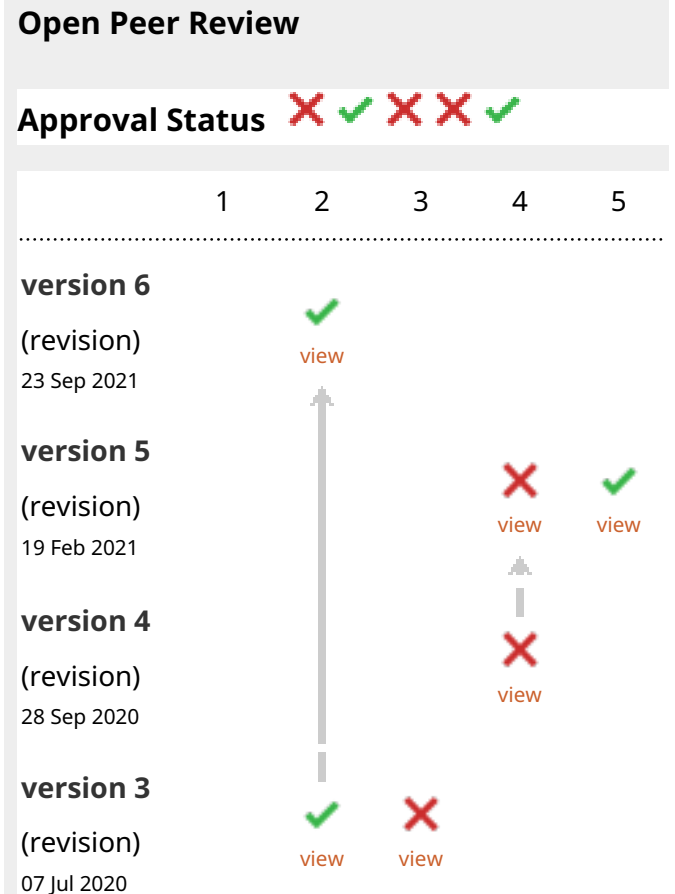

07 Jul 2020

version 2
(revision)
03 Jun 2020
version 1 X

16 Apr 2020 view

1. Indranil Banik ID, University of Bonn, Bonn, Germany

2. Marcos C.D. Neves, State University of Maringá, Maringá, Brazil 
Keywords

cosmic background radiation, cosmology theory, concordance cosmology, big bang cosmology

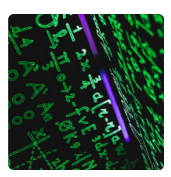

This article is included in the Mathematical, Physical, and Computational Sciences

collection.
3. Abhas Mitra ID, Bhabha Atomic Research

Centre, Mumbai, India

4. Louis Marmet (iD), York University, Toronto,

Canada

5. Matthew R. Edwards (iD), University of

Toronto, Toronto, Canada

Any reports and responses or comments on the article can be found at the end of the article.

Corresponding author: Hartmut Traunmüller (hartmut@ling.su.se)

Author roles: Traunmüller H: Conceptualization, Formal Analysis, Investigation, Methodology, Project Administration, Resources, Supervision, Validation, Visualization, Writing - Original Draft Preparation, Writing - Review \& Editing

Competing interests: No competing interests were disclosed.

Grant information: The author(s) declared that no grants were involved in supporting this work.

Copyright: $\odot 2020$ Traunmüller H. This is an open access article distributed under the terms of the Creative Commons Attribution License, which permits unrestricted use, distribution, and reproduction in any medium, provided the original work is properly cited.

How to cite this article: Traunmüller $\mathrm{H}$. Does standard cosmology really predict the cosmic microwave background? [version 1; peer review: 1 not approved] F1000Research 2020, 9:261 https://doi.org/10.12688/f1000research.22432.1

First published: 16 Apr 2020, 9:261 https://doi.org/10.12688/f1000research.22432.1 


\section{Introduction}

In 1964, Penzias \& Wilson (1965) serendipitously discovered the cosmic microwave background (CMB), a thermal radiation with a temperature of $2.7 \mathrm{~K}$. Prior to this, the presence of a cosmic heat bath with a temperature of a few $\mathrm{K}$ had already been conjectured by several researchers on various grounds unrelated to the Big Bang (Assis \& Neves, 1995). Based on absorption lines of interstellar CN-molecules, McKellar (1940) had suggested a maximum temperature of interstellar space of no more than $2.7 \mathrm{~K}$. Alpher \& Herman (1948) and Alpher et al. (1967), who were contemplating thermonuclear reactions in the expanding universe (for historical perspectives see Naselsky et al. (2006) and Alpher (2012), expected a thermal radiation with about $5 \mathrm{~K}$ as a residual of a hot Big Bang. In this, they built on Tolman's studies (Tolman, 1931; Tolman, 1934) of model universes filled with blackbody radiation as a thermodynamic fluid, so that "The model of the expanding universe with which we deal, then, is one containing a homogeneous, isotropic mixture of matter and blackbody radiation" (Alpher \& Herman, 1975). They did not really discuss and clarify under which conditions such a state is sustainable in Big Bang models.

When Penzias \& Wilson (1965) were bothered by the presence of unexpected radiation, another group of scientists (Dicke et al., 1965) did expect it in a hot Big Bang model and was developing an experiment in order to measure it. After asking whether the universe could have been filled with black-body radiation from its possible high-temperature state, they say "If so, it is important to notice that as the universe expands the cosmological redshift would serve to adiabatically cool the radiation, while preserving the thermal character. The radiation temperature would vary inversely as the expansion parameter (radius) of the universe." This is also what Tolman (1934) said.

Dicke et al. (1965) were initially in favor of a model in which the universe expands, slows down and contracts to a minimal size (not necessarily a singularity), for a new cycle to begin, but they concluded that "with the assumption of general relativity and a primordial temperature consistent with the present $3.5^{\circ} \mathrm{K}$, we are forced to adopt an open space, with very low density." (Dicke et al., 1965). They had expected the temperature to exceed $30 \mathrm{~K}$ in a closed space.

In subsequent Big Bang models, the universe expanded from a very dense and opaque initial state in which it was filled with a hot and dense plasma consisting of protons, electrons and photons colliding with these. When the plasma had cooled sufficiently by the expansion of the universe, electrons and protons combined into $\mathrm{H}$ atoms. This event is still referred to as "recombination", although cyclic models had lost support in the late 1990s, when an accelerated expansion suggested itself (within the Big Bang paradigm) in the redshift-magnitude relation of supernovae (Perlmutter, 2012; Riess, 2012; Schmidt, 2012) instead of an expected decelerated one. Only after recombination and decoupling, when the charged particles had been neutralized, the photons could move freely.

It is now commonly estimated that the universe became transparent about 380,000 years after the Big Bang (Smoot, 2007), when it had cooled to about $3000 \mathrm{~K}$. The thermal radiation is said to have been emitted from a "last scattering surface" (LSS) and to have retained its blackbody spectrum because it expanded adiabatically. Due to the ever continuing expansion, which uses to be ascribed to "space", the light waves were stretched and their energy density decreased. The wavelength at which the radiation is strongest, which according to Wien's displacement law is inversely proportional to temperature, would have become roughly 1100 times longer since the radiation was emitted (Bennet et al., 2003), while the temperature decreased to the present $2.7 \mathrm{~K}$. Since the 1970 s, the presence of this radiation has routinely been advanced as the strongest piece of evidence for a hot Big Bang.

The idea that the CMB comes directly, although redshifted, from a last scattering surface emerged only after 1965. The followers of Tolman (1934) may still have assumed that the CMB photons we receive have been further scattered on their way to us. In any case, they did not raise the questions of what constitutes the confinement of an expanding universe and which difference the motion or absence of a boundary surface would make. The problem we are concerned with here arose at the latest when these questions were still not raised when the assumption of a directly viewed LSS had made them crucial.

\section{The problem}

If one considers the following question, one can easily see that Big Bang cosmology requires the universe to be suitably confined or curved in order for radiation from the LSS to become visible at all.

If the $\mathrm{CMB}$ originated at the last scattering surface

and all matter originated within the region enclosed by this surface, while light escaped from there at $c$, maintaining this velocity for eons, and the matter of which we consist left the same region more slowly, then, how can it be that we can see the light?

With these premises, we cannot reasonably be ahead of the light. The 'flash' of light from the LSS had a substantial duration, but it must have passed our place very long ago. Now, it could only become visible at our place if the light had been reflected back to us or taken a curved return path of the right length. In a model, this needs to be specified. Before turning to the standard model, which will be shown to be inconsistent, let us first consider a non-reflective "flat" model and then briefly also reflective versions and a positively curved model.

Model 1. In a non-reflective flat Big Bang model (curvature 0), light will escape from the expanding material universe and proceed farther at velocity $c$. The material universe will be surrounded by an expanding empty region inside a spherical shell that contains radiation, perhaps also cosmic rays, but no ordinary matter. In such a universe, the conditions assumed by Tolman (1931); Tolman (1934) and presupposed by his followers are not permanently retained after last scattering. However, the belief that radiation from a past epoch, named "relic radiation" or "residual radiation", could permanently fill the whole volume of an expanding, formerly smaller universe even in the absence of a reflective boundary surface or a suitable "curvature" was 
inherent in the reasoning by Alpher \& Herman (1948); Alpher et al. (1967) and Dicke et al. (1965), and it has remained so in the more recent literature, e.g. Peebles et al. (1991) and Peebles (1993). Alpher \& Herman (1975) described their expanding universe in retrospect as "one containing a homogeneous, isotropic mixture of matter and blackbody radiation". This can and should be read as a warning against uncritical adoption, since the authors did not reason about how such a state could maintain itself over time, given the speed difference between radiation and matter. Dicke et al. (1965) stated that "The radiation temperature would vary inversely as the expansion parameter (radius) of the universe". Their calculation presupposes the radiation to fill their expanding universe permanently. Likewise, Peebles et al. (1991) wrote: "In the standard model, ... space was (and is) filled with black-body radiation, the cosmic background radiation", but the "(and is)" qualifies as a nonsequitur. Correctly and transparently reasoned,

radiation from a past epoch fills, at each instant, only the volume that is traversed by the rays or "future light cone" from that epoch.

For an origin at the LSS and no reflection, this volume is represented by the golden V-shaped band in Figure 1. The band stands for a radiation-filled shell whose thickness remains, in comoving units, constant and equal to the diameter of the LSS. The shell surrounds an expanding volume that contains no such radiation. In such a universe, the LSS will no longer be visible to anybody who has moved at $v<<c$ when more time has passed than what light needed for crossing the universe just after it had become transparent (the vertical width of the golden bands in Figure 1). The actual CMB we see now could thus not possibly have originated there.

Model 2. In a flat Big Bang universe that is surrounded by a boundary surface, light can be reflected there. Complete reflection occurs if the impedance of space becomes infinite (or zero) there. If space just loses its existence at an "edge", the impedance becomes undefined, which is problematic, but the location of the reflective surface is also problematic. In order for the CMB to become visible, the reflection must occur at a certain distance from us, within the future light cone of the LSS. If the reflection occurred at a constant distance from us, this could work in our epoch, but the CMB would not have been visible between our epoch and the time when the direct view of the LSS was lost. If the reflection formerly occurred at a smaller distance, the CMB may have been visible then, but this would have blocked any later view from a larger distance. An elaborate model that avoids this problem and/or describes a view via repetitive reflections at opposite surfaces does not appear to have been proposed.

The present standard model is in basic respects equivalent to model 2. In it, the expansion is described by the scale factor

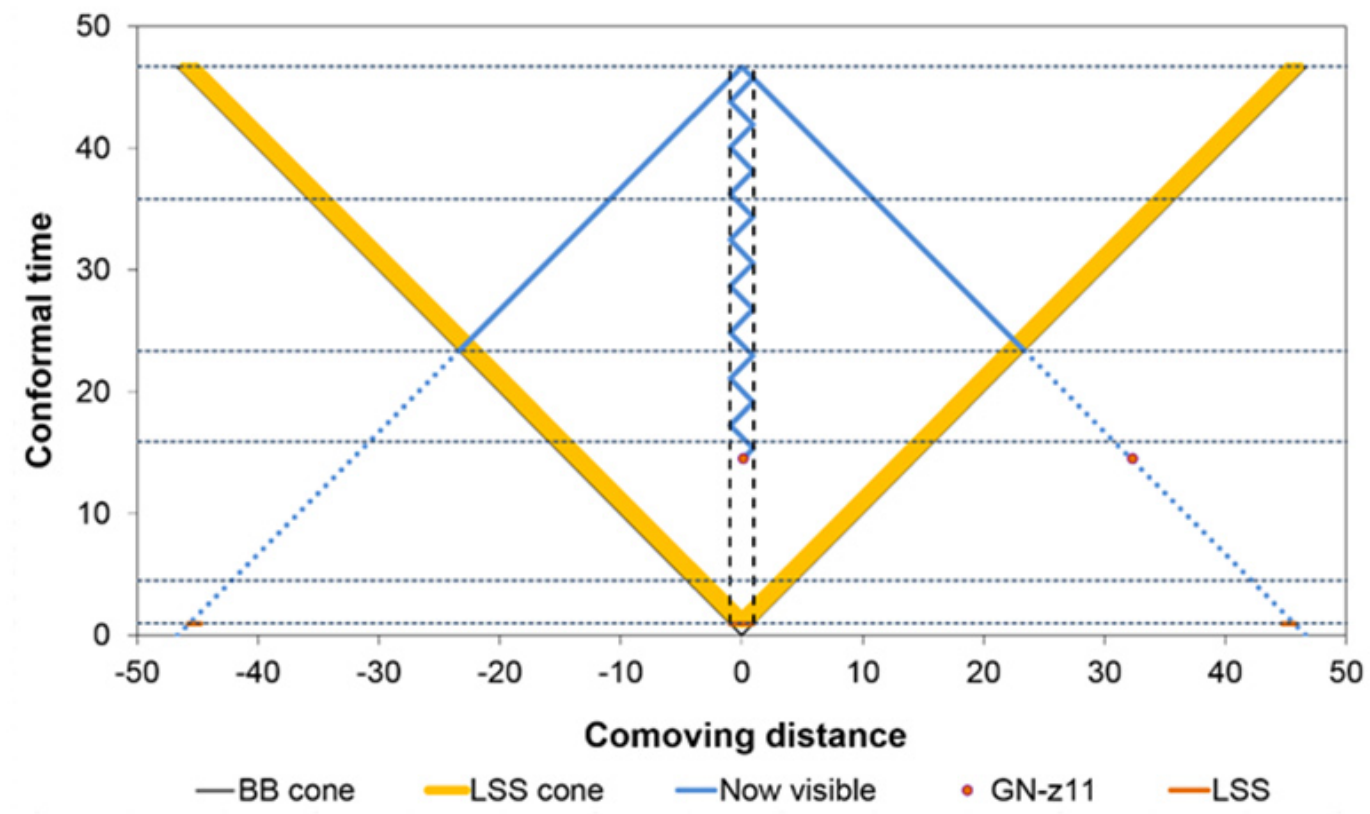

Figure 1. Spacetime diagram of flat Big Bang universe. Abscissa: comoving distance $\chi$ in Glyr. Ordinate: conformal time $\eta$ in Gyr. V-like golden band: future light cone of last scattering surface (LSS, red horizontal dash close to zero-point, visible directly only from within golden band). Blue $\Lambda$-like trace: our past light cone - we at its peak, not in golden band. Region beyond golden band (dotted extension of blue trace) has not come into existence. In standard cosmology, galaxy GN-z11 and fictitious LSS placed in this region nevertheless (the latter at $\chi \approx \pm 46$ Glyr). Between dashed vertical lines: confined universe that co-expands with material universe (co-moving diameter constant and equal to that of LSS, mentioned under model 2). So confined, LSS remains permanently visible. Place of GN-z11 in this model and zigzag path to us via 17 reflections also shown. Dotted horizontal lines: see Table 1 . Last scattering at $\eta \approx 0.95$ Gyr, $t \approx 0.38$ Myr; last visibility of LSS and blackbody conditions at $\eta \approx 1.9 \mathrm{Gyr}, t \approx 1.95 \mathrm{Myr}$. 
Table 1. Values of scale factor $a$, redshift $z$ and age $t$ of the universe, listed for conformal times $\eta$ represented by dotted horizontal lines in Figure 1.

\begin{tabular}{|c|c|c|c|c|}
\hline $\begin{array}{c}\text { Conformal } \\
\text { time } \boldsymbol{\eta}(\mathbf{G y r})\end{array}$ & $\mathbf{a}$ & $\mathbf{z}$ & $\boldsymbol{t}$ (Gyr) & Notes \\
\hline 46.7 & 1 & 0 & 13.7 & Now \\
\hline 35.8 & 0.5 & 1 & 5.95 & \\
\hline 23.35 & 0.21 & 3.76 & 1.70 & $\begin{array}{c}\text { Conformal } \\
\text { halftime }\end{array}$ \\
\hline 15.9 & 0.1 & 9 & 0.56 & \\
\hline 4.5 & 0.01 & 99 & 0.017 & \\
\hline 1.0 & 0.001 & 999 & 0.00044 & \\
\hline
\end{tabular}

Values based on 5-year WMAP data and $\Lambda$ CDM model computed using WolframAlpha ${ }^{\circledR}$

$a(t)=(1+z)^{-1}$, which is applied to co-expanding structures in three dimensions and also to the dimension of time, while

it is disregarded that radiation not only expands in these four dimensions but also escapes from its origin at $c$

and so disappears from direct view, remaining within the golden band in Figure 1. This traditional disregard is an embarrassing blunder rather than a deliberate choice. It would be permissible if the radiation lost from a region was balanced by an equal amount gained from outside, which may hold in an infinite universe, but there is no such outside to a flat Big Bang universe. It would also be justified if the material universe was surrounded by a reflective "firmament" whose diameter also expanded at $a(t)$. This diameter would, then, remain constant in units of comoving distance, which is a distance measure in which the expansion of the universe has been factored out (consider the dashed vertical lines in Figure 1). If the enclosed space in these units was as large as the LSS, it would indeed remain homogeneously filled with reflected radiation, and the CMB would evolve as traditionally assumed and taught, e.g., in Chapter 6 of Peebles' (1993) authoritative textbook. However, such a reflective firmament is for good reasons not specified in standard cosmology. It would be incompatible with the cosmological principle even in its imperfect form (which already allows violation in the dimension of time). It remains also unclear how matter that hits the firmament would interact with it.

Model 3. In a positively curved Big Bang model (curvature +1 ), which, reduced by one dimension, can be imagined as the surface of an inflating balloon, the LSS could be visible because these models allow a return path of light. This visibility can be expected to evolve with the expansion factor of the universe from continuous to periodic before finally being lost. Here, we shall not delve into the question under which premises it could be permanent or be lost entirely, because it would require assumptions that are not made in standard cosmology. Instead, when analyses of high-resolution maps of the CMB were found to be compatible with a flat universe (Davis et al., 2007; de Bernardis et al., 2000) rather than with a positively curved one, the flat universe became adopted as the standard. This flatness came unpredicted and posed a "coincidence problem" (Debono \& Smoot, 2016). Recently, based on CMB data from the Planck mission, a positive curvature has been argued for (Di Valentino et al., 2019), but this is not a feature of the present standard model.

Model 4. The present standard model (Ryden, 2016; Smoot, 2007) is a confused version of model 1 , in which the universe is flat and non-reflective, although the CMB radiation density is calculated as if the material universe was surrounded by a reflective sphere that co-expanded with the LSS, as in model 2. Despite this, which already involves the blunder mentioned there, the reasoning is further confused by accepting without further ado that the CMB we actually see originates from a different place, namely from a spherical surface or shell around the observer's, i.e., our position, from which it has taken the photons $13.7 \mathrm{Gyr}$ to reach us directly by now. This is distinctly problematic because a flat Big Bang universe in which no reflection occurs contains no points from which it would take so much time.

As can be seen in Figure 1, the points of origin are located outside the space that was brought into existence by the Big Bang, whose future light cone, from another point of view referred to as the "particle horizon", delimits this space. In this Figure, "conformal time" (presently $\eta=46.7 \mathrm{Gyr}$ ) and "comoving distance" (in Glyr) have been chosen as coordinates for the clearest illustration of the crucial aspects. Both of these coordinates are nonlinearly expanded, but they give us the same constant light cone slopes of \pm 1 lyr/yr as in a flat and static spacetime. The apparent places of origin of the $\mathrm{CMB}$, which define a fictitious LSS, are maximally remote, in comoving distance about \pm 45.7 Glyr farther away from the original LSS, at which the temperature is calculated to have been $3000 \mathrm{~K}$ at decoupling, i.e., at $t=380$ kyr. In terms of comoving distance, the extension of this surface had then already grown to almost \pm 1 Glyr, but no more than that. Note that the use of ordinary, unexpanded coordinates would make the place-discrepancy much smaller, but it would not make any difference to what is inside and outside the Big Bang universe.

The statement that it takes a CMB photon 13.7 Gyr to reach us here and now is just an estimate of the time that has elapsed since its emission. While CMB photons may actually require this time to reach us from their source, and the Universe may well be flat and infinite, a flat and reflection-free Big Bang universe does not provide the spacetime that would be necessary in order to accommodate a ray (a null-geodesic) of the corresponding length. If a ray of this length is to end at us, it must have its origin outside the Big Bang universe. This may well be so, but if this is accepted, the very idea of a Big Bang is untenable and must be rejected. It is irrational to calculate the properties of the CMB on the basis of its origin at a LSS inside a Big Bang universe and simultaneously to admit its origin at a maximally remote place outside the said universe, where the conditions are very different if ascertainable at all. The custom of referring also to this place by the attributes of "last scattering", "decoupling" etc., which apply only in an expanding universe, is deceptive. 
Figure 1 illustrates the relevance of the problem to other observables than the CMB as well: in a flat geometry,

our direct view is limited to events that happened after the universe had attained half its present age in conformal time (at $\eta \approx$ 23.35 Gyr).

This corresponds to $t \approx 1.7 \mathrm{Gyr}$, scale factor $a(t) \approx 0.21$ and redshift $z \approx 3.78$. It is noted as "conformal halftime" in Table 1 . In order for earlier events to be seen, Big Bang cosmology requires light to take a straight or curved forward and return path. This appears to have gone unnoticed by observers of distant galaxies. About GN-z11, with redshift $z=11.09$, it is reported that "This indicates that this galaxy lies at only $\sim 400 \mathrm{Myr}$ after the Big Bang" (Oesch et al., 2016), at $a(t) \approx 0.083$. This actually puts the galaxy, shown in Figure 1, far beyond the future light cone of the Big Bang. If anything exists in this spacetime region, it cannot have arrived there from the presumed ultimate origin of matter. The first galaxy that, with $z=3.8$, was too far away to be seen directly in a Big Bang universe had been observed already in 1987 (Chambers et al., 1990). If galaxies at $z>4$ cannot even be located within such a universe, it is no longer a surprise that they do not show the evolution they should according to the hierarchical merging paradigm that has become part of concordance cosmology (Steinhardt et al., 2016).

In stark contrast to what is traditionally claimed, the CMB actually tells against a formerly smaller universe and so do the most distant galaxies. The visibility of these is hard to reconcile with the idea of a Big Bang. As for the visibility of the CMB, the selfcontradiction might disappear if we actually saw mirror images [as in model 2], but in order for galaxies to be seen in this way and the actual isotropy of the CMB to be obtained, the reflector would need to be of all too spectacular stability and flatness like that required in a telescope of giga-lightyears in length. As mentioned previously, the presence of a reflective confinement would also be incompatible with the cosmological principle and put us not only at a special time but also at a special place in the universe.

\section{Discussion}

Because of the inherent inconsistency of the standard $\Lambda$ CDM concordance cosmology, here represented by model 4 , it does not come as a surprise that "misconceptions and confusions have long been common in papers on cosmology, also in many by renowned authors", as reported by Davis \& Lineweaver (2004). These authors deserve credit for having paid attention to those. However, they did not either notice that early events cannot be seen directly. In proceeding without considering reflections (last passage of their section 3.3), they mistook the intersection between our past light cone and the future light cone of the LSS [where a reflection would need to occur] for "the points from which the CMB was emitted" (Davis \& Lineweaver, 2004, p. 101). Although this is not yet beyond the particle horizon of the Big Bang, it would still be off target by half as much as model 4). The confusion arose by equating this particle horizon with the surface of last scattering, which the authors refer to as "our effective particle horizon" (Davis \& Lineweaver, 2004). It also disagrees with the caption of their Figure 1, which presupposes model 4 as such.
When Tolman (1931) considered "the highly idealized model of a non-static universe filled with black-body radiation as a thermodynamic fluid", he did not discuss the implications of the large size of the universe and the possible absence of a reflective confinement or its equivalent. It deserves to be noted that the time required for cavity radiation to attain a desired degree of homogeneity (after a sufficient number of reflections) increases in proportion to the linear size of the cavity. In a Big Bang universe, this will even with modest demands take much longer than its age. If there is no boundary surface other than one that recedes at $c$, we have seen that any old radiation will eventually disappear from view. In a flat and non-reflective Big Bang universe [model 1) above and 4) before confusion], this must happen to the radiation from the original LSS, which, thus, cannot remain visible. The CMB must have a different source, whose identification exceeds the scope of this paper.

While the irrationality of the assumption about the visibility of radiation from a past epoch in a Big Bang universe, which was disclosed in The problem, can be clearly seen in a spacetime diagram such as Figure 1, it may be missed if the ordinary coordinates of time and distance are used, especially if a past light cone is shown (in these coordinates shaped like an avocado seed) that continues below $t=1.7$ Gyr down to the origin, while it is not made evident that the region it traverses there lies outside the Big Bang universe. For examples see the "avocado seeds" in Davis \& Lineweaver (2004), more detailed in Whittle and without any scale under "Manipulating Space-Time Diagrams" in Wright.

The fact that the irrationality has remained unnoticed by professionals is an instance of the ordinary uncritical passing down of human culture, of languages, myths, etc. from generation to generation. In this wider cultural context, science stands out as an exceptional, more critical endeavor that requires practitioners not simply to accept and adopt what they were taught, but to check the relevant assumptions and doctrines for consistency and tenability and to recheck them when premises and/or relevant knowledge change. This may sometimes fail to happen, especially in cases like this, where the presence of an inconsistency became potentially clear only gradually, here after 1965, when a teaching practice had already established itself since Tolman (1934). This practice appears to have prevented the disclosure of the irrationality, which would likely have become obvious after a fresh look at the facts. It is in line with this that the rejection of the idea of a Big Bang has been blocked in model 4, although the evidence that requires the rejection has been accepted. Such blockage fosters chimera and absurd excuses ("inflation" and a plurality of universes), which have been left out of consideration here. While scientific journals often tolerate speculative ideas like these, it is unfortunate that most of them refuse through prejudice to publish any paper that discredits the currently accepted doctrine within their field from inside. For editors, it is rational to reject such papers right away: these might threat their reputation if later shown to be erroneous. Also for reviewers who lack a critical attitude against the established practice and doctrine, it is a priori inconceivable that the whole community of well-educated professionals, here mainstream cosmologists, could have made 
the same cardinal blunder. This holds also in cases like the present one, in which the presence of at least one inconsistency is obvious to the uncommitted.

Although the deficiencies disclosed here can be judged as completely unacceptable, other ones need to be addressed as well (López-Corredoira, 2017; Merritt, 2017; Spergel, 2015; Traunmüller, 2014; Traunmüller, 2018). Just consider that both $\Lambda$ (dark energy) and CDM (cold dark matter) have remained in the imaginary realm and so merely represent mythical factors or immunizing tactics (also called "conventionalist stratagems") that protect a doctrine from empirical falsification (Merritt, 2017).
Approaches that rely on such factors are at least excessively speculative, but inconsistencies such as the two revealed here must be desisted from in any discipline that is meant to qualify as rational. In order to progress, one should preferably eliminate any old deficiencies instead of suggesting some fancy new physics that might hide them. One should rather strive for well-foundedness in the physical principles (Traunmüller, 2018) than merely for a rationalized mythology, but it is, of course, even more fundamental to respect reason at all.

\section{Data availability}

No data are associated with this article.
Alpher RA, Gamow G, Herman R: Thermal Cosmic Radiation and the Formation of Protogalaxies. Proc Natl Acad Sci U S A 1967; 58(6): 2179-2186. PubMed Abstract | Publisher Full Text | Free Full Text

Alpher RA, Herman RC: On the Relative Abundance of the Elements. Phys Rev. 1948; 74(12): 1737-1742

Publisher Full Text

Alpher RA, Herman R: Big bang cosmology and the cosmic black-body radiation. Proc Am Phil Soc. 1975; 119(5): 325-348.

Reference Source

Alpher VS: Ralph A. Alpher, Robert C. Herman, and the cosmic microwave background radiation. Phys Perspect. 2012; 14: 300-334.

Publisher Full Text

Assis AKT, Neves MCD: History of the 2.7 K Temperature Prior to Penzias and Wilson. Apeiron. 1995; 2: 79-87.

Reference Source

Bennet CL, Halpern M, Hinshaw G, et al.: First-year Wilkinson Microwave Anisotropy Probe (WMAP) ${ }^{\star}$ observations: preliminary maps and basic results. Astrophys J Supp/ Series. 2003; 148: 1-27.

Publisher Full Tex

Chambers KC, Miley GK, van Breugel WJM: 4C 41.17 - A radio galaxy at a redshift of 3.8. Astrophys J. 1990; 363: 21-39.

Publisher Full Text

Davis TM, Lineweaver CH: Expanding confusion: common misconceptions of cosmological horizons and the superluminal expansion of the universe.

Publ Astron Soc Aust. 2004; 21(1): 97-109.

Publisher Full Text

Davis TM, Mörtsell E, Sollerman J, et al.: Scrutinizing exotic cosmological models using ESSENCE supernova data combined with other cosmological probes. Astrophys J. 2007; 666(2): 716-725.

Publisher Full Text

de Bernardis P, Ade PA, Bock JJ, et al: A flat universe from high-resolution maps of the cosmic microwave background radiation. Nature. 2000; 404(6781): 955-959.

PubMed Abstract | Publisher Full Text

Debono I, Smoot GF: General Relativity and Cosmology: Unsolved Questions and Future Directions. Universe. 2016; 2(4): 23.

Publisher Full Text

Dicke RH, Peebles PJE, Roll PG, et al.: Cosmic black-body radiation. Astrophys J. 1965; 142: 414-419.

Publisher Full Text

Di Valentino E, Melchiorri A, Silk J: Planck evidence for a closed Universe and a possible crisis for cosmology. Nat Astron. 2019; 4: 196-203.

Publisher Full Text

López-Corredoira M: Tests and problems of the standard model in cosmology. Found Phys. 2017; 47: 711-768.

Publisher Full Text

McKellar A: Evidence for the molecular origin of some hitherto unidentified interstellar lines. Publ Astron Soc Pac. 1940; 52: 187-192.

Publisher Full Text

Merritt D: Cosmology and convention. Stud Hist Philos Mod Phys. 2017; 57: 41-52. Publisher Full Text
Naselsky PD, Novikov DI, Novikov ID: The Physics of Cosmic Microwave Background. 2006. Cambridge University Press. Chapter 1.

Reference Source

Oesch PA, Brammer G, van Dokkum PG, et al.: A remarkably luminous galaxy at $\mathrm{z}=11.1$ Measured with Hubble space telescope grism spectroscopy. Astrophys $\mathrm{J}$. 2016; 819(2): 129.

Publisher Full Text

Peebles PJE: Principles of Physical Cosmology. Princeton University Press; Chapter 6. 1993.

Reference Source

Peebles PEJ, Schramm DN, Turner EL, et al:: The case for the relativistic hot big bang cosmology. Nature. 1991; 352: 769-776.

Publisher Full Text

Penzias AA, Wilson RW: A measurement of excess antenna temperature at 4080 Mc/s. Astrophys J. 1965; 142: 419-421.

Publisher Full Text

Perlmutter S: Nobel Lecture: Measuring the acceleration of the cosmic expansion using supernovae. Rev Mod Phys. 2012; 84(3): 1127-1149. Publisher Full Text

Riess AG: Nobel Lecture: My path to the accelerating Universe. Rev Mod Phys. 2012; 84(3): 1165-1175.

Publisher Full Text

Ryden B: Introduction to Cosmology. San Francisco: Addison Wesley; Chapters 9 and 11.2016

Reference Source

Schmidt P: Nobel Lecture: Accelerating expansion of the Universe through observations of distant supernovae. Rev Mod Phys. 2012; 84(3): 1151-1163. Publisher Full Text

Smoot GF: Nobel Lecture: Cosmic microwave background radiation anisotropies: Their discovery and utilization. Rev Mod Phys. 2007; 79: 1349-1379. Publisher Full Text

Spergel DN: The dark side of cosmology: Dark matter and dark energy.

Science. 2015; 347(6226): 1100-1102.

PubMed Abstract | Publisher Full Text

Steinhardt CLP, Capak $\mathrm{P}$, Masters $\mathrm{D}$, et al: The impossibly early galaxy problem. Astrophys J. 2016; 824: 21

Publisher Full Text

Tolman RC: On the problem of the entropy of the universe as a whole. Phys

Rev. 1931; 37: 1639-1660.

Publisher Full Text

Tolman RC: Relativity, Thermodynamics and Cosmology. Oxford: Clarendon 1934.

Reference Source

Traunmüller H: From magnitudes and redshifts of supernovae, their light-curves, and angular sizes of galaxies to a tenable cosmology. Astrophys Space Sci.

2014; 350: 755-767.

Publisher Full Text

Traunmüller H: Towards a more well-founded cosmology. Z Naturforsch A. 2018; 73: 1005-1023.

Publisher Full Text 


\section{Open Peer Review}

\section{Current Peer Review Status: $\mathrm{X}$}

Version 1

Reviewer Report 29 April 2020

https://doi.org/10.5256/f1000research.24751.r62422

(C) 2020 Banik I. This is an open access peer review report distributed under the terms of the Creative Commons Attribution License, which permits unrestricted use, distribution, and reproduction in any medium, provided the original work is properly cited.

\section{Indranil Banik}

Helmholtz Institute for Radiation and Nuclear Physics (HISKP), University of Bonn, Bonn, Germany

The following article claims to raise serious conceptual problems with the standard cosmological model:

I have to recommend that the article be rejected. First of all, the discussion section is offensive - as a researcher working on non-mainstream ideas, I can understand the sometimes difficult struggle when challenging the mainstream paradigm. But to suggest that I am recommending rejection in order to protect my career is extremely offensive, when my career in fact relies on challenging the mainstream view. The referee might like to know this before dismissing my rejection as a sign of anything other than scientific invalidity of his ideas. But I agree that it is occasionally possible for articles to be rejected which are actually correct, because the referee is protecting personal interests. This is certainly not as common as the author makes out, and indeed I have had generally respectful discussions with mainstream cosmologists despite viewing the Universe very differently. For such a (hopefully) polite discussion, the author may like to watch this debate:

\section{https://www.eso.org/sci/meetings/2020/Cosmic-Duologues.html}

Regarding the article itself, the main problem is the author has not understood the basics of the Big Bang model in which there is not an explosion in space, but an expansion of space. In this model, the universe is infinite and almost homogeneous at early times. In co-moving co-ordinates, this expansion is cancelled out and you would just see a static Universe. Suppose a flash of light is emitted from every location at the same time. Photons in some region would of course be moving at $c$, thus leaving through the boundary - but other photons would enter. The number density of photons would thus remain the same in co-moving co-ordinates. There is no inconsistency here. It explains why we expect to measure the same co-moving number density of primordial photons today as there was at the time of last scattering.

Regarding the issue of where the surface of last scattering is, the author should simply consider a photon that has been travelling at c for a Hubble time in a straight line. The result is at some distance, independent of direction - so the surface is a sphere. However, this is not a real surface - 
it is just the locus of points from which photons will later hit the Earth exactly 13.8 Gyr later. At the time of emission, nothing whatsoever is special about material in this surface. The whole sphere may well be much larger than $380 \mathrm{kly}$, which is somewhat non-intuitive since the age of the universe was (in this model) only $380 \mathrm{kyr}$ then.

The author raises an important point about how the Universe was in thermal equilibrium at early times. This is related to the horizon problem, which - as the author points out - is thought to be resolved by inflation. Briefly, the idea is that the Universe was small for an extended period of time, during which it was in causal contact and thus reached thermal equilibrium. The particle horizon then expanded faster than c due to a period of accelerated expansion similar to what we are experiencing now, causing that photons reaching us from different sides of the sky have the same temperature. The author then goes on to unscientifically attack the hypothesis of inflation indeed, it is well known that this is not confirmed. However, the author does not raise any substantive arguments against the inflation model, and does not propose his own ideas.

Of course, if the mainstream idea was that the Universe was 380 kly wide at some time $13.8 \mathrm{Gyr}$ ago and that every location within it emitted photons at that moment (almost) equally in all directions, then it is clear that these photons would be unobservable now - depending on what happens at the edge. However, the Universe is not thought to have any such edge, and is considered to be infinite. It is certainly the case that light emitted from close to us would no longer be observable, so what we see as the cosmic microwave background (CMB) must have originated rather far away, on the surface of last scattering. The longer we wait, the further away this surface is - though it always corresponds to the same moment in the history of the universe. There will never be a moment when there is no CMB altogether (barring absorption of all its photons), because one can always imagine reversing the arrow of time and integrating a trajectory moving away from us at $\mathrm{c}$ in a contracting universe. The photon will have some well-defined location at the moment of last scattering, so this point will be part of the surface of last scattering to such an observer. No special reflective surface is required, and indeed no new assumptions are needed at all to explain the observability of the CMB. Of course, explaining its detailed properties remains a challenge given other constraints e.g. the Hubble tension. But the particular criticism of the standard cosmological model raised by the author is completely erroneous. Therefore, this manuscript must be rejected - even though I do consider that the same is true for the standard cosmological model as a whole, which does indeed contain many hypothetical ingredients that I am not at all a fan of \& have publicly spoken against on several occasions e.g. here:

https://www.youtube.com/watch?v=PYVCOVtmpDg

Is the topic of the opinion article discussed accurately in the context of the current literature?

Yes

Are all factual statements correct and adequately supported by citations?

No

Are arguments sufficiently supported by evidence from the published literature? No

Are the conclusions drawn balanced and justified on the basis of the presented arguments? 
Competing Interests: No competing interests were disclosed.

Reviewer Expertise: Modified Newtonian Dynamics (MOND) I felt I was able to assess all aspects of the article, as I work on a non-mainstream cosmological model and need to pay close attention to issues like the cosmic microwave background radiation and cosmology, which remains a little uncertain in MOND

I confirm that I have read this submission and believe that I have an appropriate level of expertise to state that I do not consider it to be of an acceptable scientific standard, for reasons outlined above.

Author Response 14 May 2020

Hartmut Traunmüller, Stockholm University, Stockholm, Sweden

Reviewer's comment: I have to recommend that the article be rejected. First of all, the discussion section is offensive - as a researcher working on non-mainstream ideas, I can understand the sometimes difficult struggle when challenging the mainstream paradigm. But to suggest that I am recommending rejection in order to protect my career is extremely offensive, when my career in fact relies on challenging the mainstream view. The referee might like to know this before dismissing my rejection as a sign of anything other than scientific invalidity of his ideas.

Author's response: This review (there is no separate referee) emphasizes something other on the preceding lines. It has probably to do with my mentioning, in the next to last passage of the article, of a good reason editors might have for rejecting certain manuscripts prior to review. In the same passage, I suggest a milder, equally rational explanation - one that applies beside editors also to reviewers and definitely also to myself in many analogous situations.

Reviewer's comment: But I agree that it is occasionally possible for articles to be rejected which are actually correct, because the referee is protecting personal interests. This is certainly not as common as the author makes out,

Author's response: This is common in certain cases, also self-censorship. To make it clear in which cases, I have inserted a reference to Lakatos, who had observed that the "hard core" of "research programmes" (such as mainstream cosmology) is beyond criticism.

Reviewer's comment: the main problem is the author has not understood the basics of the Big Bang model in which there is not an explosion in space, but an expansion of space.

Author's response: In the next to last passage of the Introduction, in which I summarize the characteristics of a Big Bang model that are essential here, I told that the expansion uses to be ascribed to "space", i.e., to an expansion of space. An expansion in space was nowhere implied, but at the end of the second passage under Model 4, I have now inserted a 
parenthetic phrase "or at least empty" after "non-existent" only for telling that this related distinction makes no difference in this context.

Author's response to the Reviewer's remaining comments: These have brought me to underline the fact that, in a Big Bang universe, it is not the case that blackbody radiation lost from a region is balanced by an equal amount gained from outside and, above all, to extend the description and analysis of the present standard model (new text under Model 4) as summarized in the "Update text". I kindly ask the reviewer for a new evaluation.

Competing Interests: No competing interests were disclosed.

The benefits of publishing with F1000Research:

- Your article is published within days, with no editorial bias

- You can publish traditional articles, null/negative results, case reports, data notes and more

- The peer review process is transparent and collaborative

- Your article is indexed in PubMed after passing peer review

- Dedicated customer support at every stage

For pre-submission enquiries, contact research@f1000.com 